\title{
A Review on Weed Detection Using Image Processing
}

\author{
Lavanya N.R.*, Niharika S., Deepika C.H., Harini M. and Chetana K.S. \\ Department of Electronics and Communication Engineering, Vidyavardhaka College of Engineering, Mysuru, India; \\ lavanya03398@gmail.com,nihariaru16@gmail.com, deepikahgowda87@gmail.com,mharinimys@gmail.com, \\ chethanaks@vvce.ac.in
}

\begin{abstract}
Objectives: To detect and map the specific weed to control the cost of farming, and enhance productivity. Methods analysis: There are various methods used for weed detection. Image processing methods are used along with artificial intelligence methods like ANN (Artificial neural networks) and Support Vector Machine (SVM) to identify the weed. Findings/application: Weeds are identified from the image, making use of image processing technique by shape, color and size feature using Support Vector Machine or Artificial Neural Network. Since hand labor is expensive and automatic weed controlling would be more feasible and the robotic weed controller can decrease or eliminate the need for chemicals.
\end{abstract}

Keywords: Weed Detection, Image Processing, Artificial Neural Network, Support Vector Machine

\section{Introduction}

A weed is a plant that is considered as unwanted in a particular situation, "a plant in the wrong place". Weed identification is the identification of weed or undesirable plants from desirable plants in the same field. Various criteria are used for identifying weed from the desired plant. Image processing is one of the technologies used in the same task.

Weed identified from images using image processing technique is differentiated by shape, color and size features. These characteristics are used to classify different weeds and crop species. Image processing algorithms are used to take images of the plantation rows at regular intervals of time. If the weed is detected robots are used to spray herbicides. Weeds are identified also by the probabilistic neural networks. Otsu's technique is used for the automatic threshold for segments of weeds. Weed is identified using angular cross-section intensities, for high accuracy support vector machines (SVM) can be used.

The detection and classification of weeds are of major technical and economic importance in the agricultural industry. Identification of weed and extraction of it increases productivity on feature large scale and therefore leading to better income. Weed identification at the early stage can prevent the desired plants from getting effected. Thus detection and classification of weeds prove to play an important role in agriculture.

\section{Literature Survey}

Weeds compete with the crop for sunlight as well as for the required nutrients and resources from the soil. 1 Effective removal of weeds improves the productivity of the crops. Identification and removal of weeds is a challenging task. Current weed removal methods use mechanical, 2 electrocuting $\underline{\underline{3}}$ or chemical 1 methods which are laborious, expensive, and toxic to the environment. An automated weed control system using a machine vision algorithm can be effective. An automated weed control system using a machine vision algorithm to identify the weeds has been developed for the automatic spraying control systems. $\underline{4}$

In $n$ used a dilation segmentation algorithm for weed recognition. This method is used to classify between narrow and broad weeds. So using the binary image processing method; i.e. (color image is converted to greyscale and then using binary image weed is identified). This algorithm was tested on sample images of 100 narrow and 100 broad leaf weeds and 40 leaf images that are not

${ }^{*}$ Author for correspondence 
weeds. This method gave an average reliable accuracy of $89 \%$ to detect the presence or absence of weed cover.

A site-specific weed management strategy was developed, where density and composition of the weed are taken into consideration. $\underline{6}$ This strategy is precise and uses resources efficiently. In this strategy, a weed map is created and weed detection is done using ground or remote sensing applications. The ground-based methods can get high-resolution images that help in detecting weeds with low densities, $\underline{\underline{Z}}$ whereas remote sensing methods help in detecting weeds in a large area but with low image resolution. .8 Recently drones using higher image resolution cameras, and powerful computers which can quickly process large data have helped in making the remote sensing method precise and accurate. ${ }^{9}$ However these methods are very expensive to use.

Low-cost RGB cameras can acquire images with good resolution and identify the plant-based on the shape, colour, texture that etc. Segmentation of the image will differentiate the soil and plant cover. The white regions in the image represent the plant and the black regions are the soil. The plant species can be identified using the shape of the leaves. Image analysis software is used to identify the shape of the crops and weeds. 10 Both narrow and broad leaf weed species are identified and an image database is created. An accuracy of $90 \%-98 \%$ was achieved in shape identification of the leaves. This method worked very well in the early growth stage of the weed. At later stages leaf overlap of the plant and the weed made it look indistinguishable after segmentation. This is a major drawback of this method.

Weeds grow in a field in different patterns, like sometimes an entire patch of the field may be covered by the weed, while otherwise, the weed may grow in between the crops. In the case of large weed patches, ground-based or remote sensing-based methods can easily identify the weeds and appropriate action can be taken. Whereas if the weed is growing in between the crops then the problem is more challenging. The remote sensing methods mostly cannot identify weeds in this situation. Ground-based methods can identify but the accuracy level is quite low. In the early growth stage of the weed, the ground-based methods like fitting a camera to the tractor and scanning the field can give satisfactory results. $\underline{11}$ Although groundbased systems using ultra-high-resolution cameras can detect individual weeds and also identify the type of plant or weed, problems still persist for accurate identification in variable illumination and in case the plant and weed leaves are overlapping. $\underline{12}$ Processing times of the obtained data is also a challenge for real-time application of these methods. Some of these challenges can be overcome by using deep learning algorithms to accurately verify the type of plants and faster analysis of the data.

Applying machine vision techniques to the captured images from the camera several features can be extracted which is useful for proper identification of weed or plant. $\underline{13}$ Some of the features that can be extracted are texture, shape and colour. When the colour of the weed is distinct from the main crop the weed and the crop can be easily distinguished. Tang et al achieved an accuracy of $92.5 \%$ in identifying weeds with distinct colours. $\underline{14}$ When the shape of the individual leaf is distinct from the crop leaf again it is easy to discriminate the images of weed and crop unless there is an overlapping of the leaves. $\frac{15}{\text { The }}$ texture is another feature that can be used to differentiate the weed and crop if the leaves are not overlapping. 16

In most cases, the weed and crop may not have distinguishing features and it is extremely difficult to distinguish the weed and the crop using a single feature. It is necessary to develop techniques that can look at all the available distinguishing features. Pattern recognition techniques such as Artificial Neural Networks (ANN) and Support Vector Machine (SVM) can combine several such features to distinguish the weed or crop. Neural networks with unsupervised $\mathrm{K}$-means feature learning as pre-training achieved an accuracy of $92.89 \%$ for weed detection in soybean.17 Based on statistical learning theory, Support Vector Machine (SVM) is a supervised learning technique.SVM was used to successfully classify chilli and weed from digital images. $\underline{18}$

Agricultural robots can be used to remove the weeds from the farm by spraying herbicide on the weeds. The image captured by the camera on the robot is analyzed using an image processing algorithm, upon identifying the weed herbicides are sprayed on the weed plants, without harming the actual crop. The image processing algorithm uses an erosion and dilation segmentation approach to detect the weeds. Using this algorithm the color image is converted into a binary image and the plant having higher white pixels than the predefined threshold is considered as a weed.

\section{Discussion}

Different techniques to identify the difference between crop and weed have been studied. Each and every 
technique has several advantages, disadvantages and limitations for it. Pattern recognition methods like artificial neural networks, support vector machines were applied to identify weeds images. Based on the shape and texture, the algorithm could identify the plant from the background as well as whether the crop and weed were overlapping or not. The artificial neural network classifier was created for the recognition of crop and weeds according to the shape features. An image processing algorithm can be used to take images of the plants and upon identifying the weeds in the image a robot can spray the herbicide directly on the weeds. After studying all the characteristics of classification techniques the Artificial Neural Network is found to be robust and suited for complex as well as incomplete data. Image processing is a non-invasive and effective tool that can be applied to detect and identify the weeds on the farm and effectively remove them.

\section{Conclusion}

The main objective is to detect and map specific weed in order to control the expenses in farming and to enhance productivity. There are various methods used for weed detection. Some of the methods are ANN(Artificial neural network) which is examined. Using an image processing algorithm, weeds are identified from the images by shape, color, texture and size features. Using the automatic weed detection the weeds can be controlled by smart herbicide spraying robots. Since hand labor is getting expensive day by day, an automatic weed control system would be feasible. A robotic weed controller can also reduce or eliminate the need for harmful chemicals.

\section{Acknowledgement}

The authors express gratitude to Accendere Knowledge Management Services Pvt Ltd for the assistance provided in preparing the manuscript.

\section{References}

1. Slaughter DC, Giles DK, Downey D. Autonomous robotic weed control systems: a review. Comput Electron Agric. 2007;61(1):63-78.

2. Pannacci E, Lattanzi B, Tei F. Non-chemical weed management strategies in minor crops: a review. Crop Prot. 2017;96:44-58.
3. Diprose MF, Benson FA. Electrical methods of killing plants. J Agric Eng Res. 1984;30:197-209.

4. Slaughter DC, Giles DK, Fennimore SA, Smith RF. Multispectral machine vision identification of lettuce and weed seedlings for automated weed control. Weed Technol. 2008;22(2):378-84.

5. Siddiqi $\mathrm{MH}$, Ahmad I, Sulaiman S. Weed recognition based on erosion and dilation segmentation algorithm. In: International conference on education technology and computer; 2009.

6. Wiles JL. Beyond patch spraying: site-specific weed management with several herbicides. Precis Agric. 2009;10(3):277-90.

7. Peteinatos G, Weis M, Andujar D. Potential use of groundbased sensor technologies for weed detection. Pest Manag Sci. 2014;70(2):190-99.

8. Rasmussen J, Nielsen J, Garcia-Ruiz F. Potential uses of small unmanned aircraft systems (UAS) in weed research. Weed Res. 2013;53(4):242-8.

9. Van Evert FK, Fountas S, Jakovetic D. Big data for weed control and crop protection. Weed Res. 2017;57(4):218-33.

10. Weis M, Gerhards R. Feature extraction for the identification of weed species in digital images for the purpose of sitespecific weed control. In: Stafford JV, editor. Precision agriculture 007. Wageningen Academic Publishers; 2007.

11. Kunz C, Weber J, Peteinatos G, Sokefeld M, Gerhards R. Camera steered mechanical weed control in sugar beet, maize and soybean. Precis Agric. 2018;19(4):708-20.

12. Ali A, Streibig JC, Christensen S, Andreasen C. Imagebased thresholds for weeds in maize fields. Weed Res. 2015;55(1):26-33.

13. Zhang J, Song F, Tang J. Identification of crop weed based on image texture features. Comput Model New Technol. 2014;18(10):203-06.

14. Tang JL, Chen XQ, Miao RH, Wang D. Weed detection using image processing under different illumination for site-specific areas spraying. Comput Electron Agric. 2016;122:103-11.

15. Kazmi W, Garcia-Ruiz FJ, Nielsen J, Rasmussen J, Andersen HJ. Exploiting affine invariant regions and leaf edge shapes for weed detection. Comput Electron Agric. 2015;118:290-99.

16. Bakhshipour A, Jafari A, Nassiri SM, Zare D. Weed segmentation using texture features extracted from wavelet sub-images. Bio-Syst Eng. 2017;157:1-12.

17. Tang J, Wang D, Zhang Z, He L, Xin J, Xu Y. Weed identification based on $\mathrm{K}$ means feature learning combined with convolutional neural network. Comput Electron Agric. 2017;135:63-70.

18. Ahmed F, Al-Mamun HA, Bari AH, Hossain E, Kwan P. Classification of crops and weeds from digital images: a support vector machine approach. Crop Prot. 2012;40:98-104. 